\title{
Apolipoprotein A-I Crosses the Blood-Brain Barrier through Clathrin-Independent and Cholesterol-Mediated Endocytosis
}

\author{
Andrew L. Zhou, Suresh K. Swaminathan, Geoffry L. Curran, Joseph F. Poduslo, \\ Val J. Lowe, Ling Li, and Karunya K. Kandimalla
}

Department of Pharmaceutics and Brain Barriers Research Center (A.L.Z., S.K.S., K.K.K.) and Department of Experimental and Clinical Pharmacology (L.L.), College of Pharmacy, University of Minnesota, Minneapolis, Minnesota; and Department of Radiology (G.L.C., V.J.L.) and Department of Neurology (G.L.C., J.F.P.), Mayo Clinic College of Medicine, Rochester, Minnesota

Received October 22, 2018; accepted March 11, 2019

\section{ABSTRACT}

Recent studies suggest that apolipoprotein A-I (ApoA-I), the major protein constituent of high-density lipoprotein particles, plays a critical role in preserving cerebrovascular integrity and reducing Alzheimer's risk. ApoA-I present in brain is thought to be primarily derived from the peripheral circulation. Although plasma-to-brain delivery of ApoA-I is claimed to be handled by the blood-cerebrospinal fluid barrier (BCSFB), a contribution by the blood-brain barrier (BBB), which serves as a major portal for protein delivery to brain, cannot be ruled out. In this study, we assessed the permeability-surface area product (PS) of radioiodinated ApoA-I $\left({ }^{125} \mathrm{I}-\mathrm{ApoA}-\mathrm{I}\right)$ in various brain regions of wild-type rats after an intravenous bolus injection. The PS value at the cortex, caudate putamen, hippocampus, thalamus, brain stem, and cerebellum was found to be $0.39,0.28,0.28,0.36$, 0.69 , and $0.76\left(\mathrm{ml} / \mathrm{g}\right.$ per second $\left.\times 10^{-6}\right)$, respectively. Solutes delivered into brain via the BCSFB are expected to show greater accumulation in the thalamus due to its periventricular location. The modest permeability for ${ }^{125} \mathrm{I}-\mathrm{ApoA}-\mathrm{I}$ into the thalamus relative to other regions suggests that BCSFB transport accounts for only a portion of total brain uptake and thus BBB transport cannot be ruled out. In addition, we show that Alexa Flour 647-labeled ApoA-I (AF647-ApoA-I) undergoes clathrin-independent and cholesterol-mediated endocytosis in transformed human cerebral microvascular endothelial cells (hCMEC/D3). Further, Z-series confocal images of the hCMEC/D3 monolayers and Western blot detection of intact ApoA-I on the abluminal side demonstrated AF647-ApoA-I transcytosis across the endothelium. These findings implicate the BBB as a significant portal for ApoA-I delivery into brain.

\section{Introduction}

Apolipoprotein A-I (ApoA-I) serves as the major protein constituent of high-density lipoprotein (HDL) particles in plasma. HDL is involved in the reverse transport of cholesterol from peripheral tissues to liver and thereby plays a critical role in protecting against hypercholesterolemia, which causes atherosclerosis (Assmann and Gotto, 2004). In addition, emerging evidence suggests that ApoA-I/HDL impacts cerebrovascular accumulation of amyloid beta $(\mathrm{A} \beta)$ peptides in Alzheimer's disease brain (Hottman et al., 2014; Stukas et al., 2014b). This has implications for the effective treatment of Alzheimer's disease (AD), in which cerebrovascular $\mathrm{A} \beta$ deposition was shown to substantially augment cognitive decline and $\mathrm{AD}$ progression (Weller et al., 2009).

This work was supported by the Minnesota Partnership for Biotechnology and Medical Genomics [Grant 00056030] and by the National Institutes of Health [Grants AG058081, AG056025].

https://doi.org/10.1124/jpet.118.254201.
Specific studies in APP/PS1 transgenic mice, which express excess $\mathrm{A} \beta$, have revealed the potential role of ApoA-I in protecting against cerebrovascular $\mathrm{A} \beta$ deposition, cerebral amyloid angiopathy (CAA), and cognitive decline. Deletion of ApoA-I was shown to increase CAA and exacerbate cognitive impairment in APP/PS1 mice (Lefterov et al., 2010). Conversely, ApoA-I overexpression was shown to reduce CAA and preserve cognitive function in APP/PS1 mice (Lewis et al., 2010). It has also been reported that luminal ApoA-I can mobilize the abluminal efflux of $\mathrm{A} \beta$ across cerebrovascular endothelial cell monolayers (Merino-Zamorano et al., 2016). Thus, ApoA-I appears to be important in alleviating $\mathrm{A} \beta$ load in the cerebral vasculature and protecting against cognitive deficits associated with $\mathrm{AD}$. As such, alterations in ApoA-I disposition and function are expected to have a significant impact on the cerebrovascular contributions to $\mathrm{AD}$ pathogenesis.

ApoA-I is the second most abundant lipoprotein after apolipoprotein $\mathrm{E}(\mathrm{ApoE})$ in the cerebrospinal fluid (Roheim et al., 1979; Koch et al., 2017), yet ApoA-I mRNA has not been detected in the brain, at least in murine models (Elliott et al., 2010).

ABBREVIATIONS: AD, Alzheimer's disease; AF647, Alexa Fluor 647; ApoA-I, apolipoprotein A-I; ApoE, apolipoprotein; AUC, area under the plasma concentration versus time curve; $A \beta$, amyloid beta; BBB, blood-brain barrier; BCSFB, blood-cerebrospinal fluid barrier; CAA, cerebral amyloid angiopathy; DAPI, 4',6-diamidino-2-phenylindole; DPBS, Dulbecco's phosphate-buffered saline; FITC, fluorescein isothiocyanate; hCMEC/D3, human cerebral microvascular endothelial cells; HDL, high-density lipoprotein; M $\beta$ CD, methyl- $\beta$-cyclodextrin; PFA, paraformaldehyde; PS, permeability-surface area product; siRNA, small interfering RNA; SR-B1, scavenger receptor class B type 1; TBST, Tris-buffered saline containing $0.1 \%$ Tween 20 ; TRF, transferrin; $V_{\mathrm{p}}$, cerebrovascular volume. 
While ApoE is synthesized both in the brain and in the periphery, ApoA-I is produced primarily by the liver and intestine. Hence, the pool of ApoA-I in the brain is thought to be delivered from the periphery through the blood-cerebrospinal fluid barrier (BCSFB) and/or the blood-brain barrier (BBB). Both barriers show exquisite selectivity in their permeability, and together they represent the two major portals regulating the flux of macromolecules between the blood and brain (Strazielle and Ghersi-Egea, 2013).

The BCSFB is lined by the choroid epithelium at the four ventricles, whereas the BBB is lined by the diffuse network of brain microvessels that comprise the cerebrovascular endothelium. Stukas et al. (2014a) have previously demonstrated the localization of Alexa Fluor 647-labeled recombinant ApoA-I (AF647-rApoA-I) at the choroid plexus after intravenous injection in mice, thereby concluding preferential ApoA-I brain entry via the BCSFB. However, the investigators noted that contribution of the BBB in ApoA-I brain delivery remains unresolved and as such warrants further investigation. Given the emerging role of ApoA-I in reducing $\mathrm{A} \beta$ deposition in the cerebral vasculature, there is a need to clarify the relevance of the BBB as a portal for ApoA-I brain delivery.

\section{Materials and Methods}

Animals. Wild-type Sprague-Dawley male rats, 16 weeks old, were obtained from Harlan Sprague-Dawley (Indianapolis, IN) and maintained in the animal facility at the Mayo Clinic. The animals were provided with food and water ad libitum. All procedures involving animals were carried out in accordance with the Guide for the Care and Use of Laboratory Animals outlined by the National Institutes of Health and were approved by the Mayo Institutional Animal Care and Use Committee.

Radioiodination of ApoA-I. Human serum-derived ApoA-I (EMD Millipore, Burlington, MA) was labeled with ${ }^{125} \mathrm{I}$ or ${ }^{131} \mathrm{I}$ radionuclides (PerkinElmer Life and Analytical Sciences, Boston, MA) using the chloramine-T procedure described previously elsewhere (Poduslo et al., 1994; Kandimalla et al., 2005). After dialysis in 0.01 M Dulbecco's phosphate-buffered saline (DPBS) to remove unconjugated radionuclide, the intact radiolabeled peptides $\left({ }^{125} \mathrm{I}-\mathrm{ApoA}-\mathrm{I}\right.$ and ${ }^{131}$ I-ApoA-I) were assayed by trichloroacetic acid precipitation.

${ }^{125}$ I-ApoA-I Brain Permeability Studies. Each rat was anesthetized with $1.5 \%$ isoflurane in $4 \mathrm{l} / \mathrm{min}$ oxygen, and the femoral vein as well as artery were catheterized. An intravenous bolus injection of ${ }^{125} \mathrm{I}-\mathrm{ApoA}-\mathrm{I}(50 \mu \mathrm{Ci})$ was administered via the femoral vein at $t=0$ minutes, and a $20 \mu \mathrm{l}$ blood sample was collected from the femoral artery at $0.25,1,5$, $10,15,30,45$, and 59 minutes after injection. To measure the residual plasma volume $\left(V_{\mathrm{p}}\right)$, an intravenous bolus injection of ${ }^{131} \mathrm{I}$-ApoA-I $(50 \mu \mathrm{Ci})$ was administered at $t=59$ minutes. A final blood sample was collected at $t=60$ minutes, and the animal was euthanized.

The blood samples were diluted to $100 \mu \mathrm{l}$ in saline and centrifuged. The supernatant was trichloroacetic acid precipitated and analyzed for intact ${ }^{125} \mathrm{I}$-ApoA-I and ${ }^{131} \mathrm{I}$-ApoA-I using a two-channel gamma counter (Cobra II; Amersham Biosciences, Piscataway, NJ). Activity counts were corrected for background and spillover between ${ }^{125} \mathrm{I}$ and ${ }^{131}$ I channels.

The brain was removed at the end of the experiment, dissected into various anatomic regions (cortex, caudate putamen, hippocampus, thalamus, brain stem, and cerebellum), and then assayed for ${ }^{125}$ I-ApoA-I and ${ }^{131}$ I-ApoA-I radioactivity. The entire experimental procedure is outlined in Fig. 1.

Cell Culture. The immortalized human cerebral microvascular endothelial cell line (hCMEC/D3) was a gift from P-O Couraud (Institut Cochin, France). The cells were cultured as described elsewhere (Weksler et al., 2013) in endothelial basal medium-2

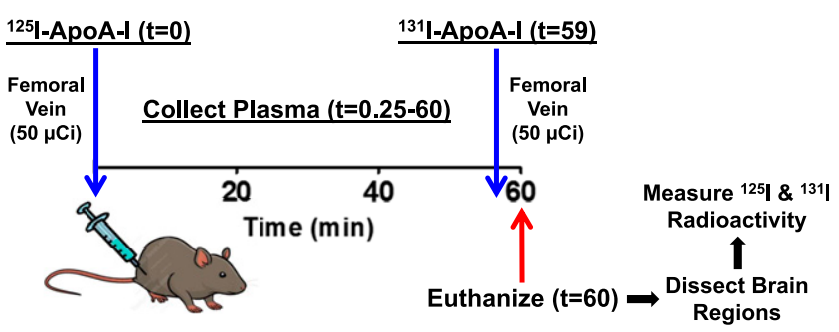

Fig. 1. Layout of studies conducted to evaluate ${ }^{125}$ I-ApoA-I plasma kinetics and brain permeability in rats.

(Lonza, Basel, Switzerland) prepared with the following additives: $1 \% \mathrm{v} / \mathrm{v}$ penicillin-streptomycin (Sigma-Aldrich, St. Louis, MO), $1.4 \mu \mathrm{M}$ hydrocortisone (Sigma-Aldrich), $5 \mu \mathrm{g} / \mathrm{ml}$ ascorbic acid (Sigma-Aldrich), $1 \% \mathrm{v} / \mathrm{v}$ chemically defined lipid concentrate (ThermoFisher Scientific, Waltham, MA), $10 \mathrm{mM}$ HEPES (Sigma-Aldrich), and $1 \mathrm{ng} / \mathrm{ml}$ recombinant human fibroblast growth factor-basic (PeproTech, Rocky Hill, NJ). The medium supplemented with these additives is hereinafter referred to as D3 medium. Cells were cultured in D3 medium containing 5\% v/v FBS (Atlanta Biologicals, Flowery Branch, GA).

Conjugation of ApoA-I with Alexa Fluor 647. Human serumderived ApoA-I was buffer exchanged into DPBS using an ultrafiltration unit with $10 \mathrm{kDa}$ molecular weight cutoff (EMD Millipore). Alexa Fluor 647 (AF647) was conjugated to ApoA-I using the labeling kit with minor deviations from the manufacturer's protocol (Invitrogen, Carlsbad, CA). Briefly, $1 \mathrm{M}$ sodium bicarbonate solution in distilled water was added to $2 \mathrm{mg} / \mathrm{ml}$ ApoA-I solution to increase the $\mathrm{pH}$ to $~ 8.0$. Reactive dye was added to the protein solution and stirred at room temperature for 1 hour. Then, fluorescently labeled ApoA-I was separated from the unconjugated dye using a dye-removal column (ThermoFisher Scientific), and the concentration of labeled protein was determined as follows:

Protein concentration $(\mathrm{M})=\frac{\left[A_{280}-\left(A_{600} \times 0.03\right)\right] \times \text { Dilution factor }}{37,410 \mathrm{M}^{-1} \mathrm{~cm}^{-1}}$

where $A_{280}$ is the absorbance of ApoA-I solution at $280 \mathrm{~nm}$ wavelength, $A_{600}$ is the absorbance at $600 \mathrm{~nm}, 0.03$ is a correction factor for dye absorption at $280 \mathrm{~nm}$, and $37,410 \mathrm{M}^{-1} \mathrm{~cm}^{-1}$ is the molar extinction coefficient of ApoA-I. The absorbance was measured using quartz cuvettes in a UV/Vis spectrophotometer (BioPhotometer 6131; Eppendorf, Hamburg, Germany). The degree of labeling was determined as follows:

\section{Moles of dye per mole of protein}

$$
=\frac{A_{600} \times \text { Dilution factor }}{239,000 \mathrm{M}^{-1} \mathrm{~cm}^{-1} \times \text { Protein concentration }(\mathrm{M})}
$$

where $239,000 \mathrm{M}^{-1} \mathrm{~cm}^{-1}$ is the molar absorptivity of AF647 dye.

Flow Cytometry Studies. The hCMEC/D3 cells were cultured in six-well plates as described elsewhere (Swaminathan et al., 2017). Upon reaching confluency, the cells were incubated for 1 hour at $37^{\circ} \mathrm{C}$ with $1 \mathrm{ml}$ of D3 medium containing $1 \% \mathrm{v} / \mathrm{v} \mathrm{FBS}$ with or without $10 \mathrm{mM}$ methyl- $\beta$-cyclodextrin $(\mathrm{M} \beta \mathrm{CD}$ ) (Acros Organics, Morris Plains, $\mathrm{NJ}$ ) or $50 \mu \mathrm{M}$ nystatin (Sigma-Aldrich). The AF647-ApoA-I $(0.4 \mu \mathrm{M})$ was added to the wells, and the plate was gently rocked to ensure even dispersion of the labeled protein. After 1 hour of incubation at $37^{\circ} \mathrm{C}$, the cells were washed twice with DPBS, trypsinized, and resuspended in $250 \mu \mathrm{l}$ of DPBS. A $250 \mu \mathrm{l}$ aliquot of ice-cold $4 \% \mathrm{v} / \mathrm{v}$ paraformaldehyde (PFA) was then added, and the cells were fixed on ice for 15 minutes. The fluorescence uptake was measured using a LSR-II Fortessa flow cytometer equipped with a $40 \mathrm{~mW}(640 \mathrm{~nm})$ laser (BD Biosciences, San Jose, CA) and analyzed using FlowJo software (TreeStar Inc., San Carlos, CA). 
Confocal Microscopy Studies. The hCMEC/D3 cells were cultured on 35-mm coverslip bottom dishes as described elsewhere (Swaminathan et al., 2017). Upon reaching confluency, the cell monolayers were preincubated for 1 hour at $37^{\circ} \mathrm{C}$ in $500 \mu \mathrm{l}$ of Dulbecco's modified Eagle's medium ( $0.1 \%$ w/v bovine serum albumin) with or without $10 \mathrm{mM} \mathrm{M} \beta \mathrm{CD}$. Then, AF647-ApoA-I $(0.4 \mu \mathrm{M})$ was added to the dish upon gentle rocking. After 1 hour of incubation at $37^{\circ} \mathrm{C}$, the cells were washed 3 times with DPBS and fixed in $1 \mathrm{ml}$ of icecold $4 \% \mathrm{v} / \mathrm{v}$ PFA on ice for 1 hour. The fixed cells were washed 3 times with DPBS, mounted with ProLong Diamond Mounting medium containing 4',6-diamidino-2-phenylindole (DAPI) (Invitrogen), and then imaged using a Zeiss LSM 780 laser confocal microscope equipped with a C-Apochromat $40 \times / 1.2 \mathrm{~W}$ objective.

For small interfering RNA (siRNA) knockdown studies, hCMEC/D3 cells were grown to confluency on 35-mm coverslip bottom dishes and transfected with clathrin heavy chain siRNA (Dharmacon, Lafayette, $\mathrm{CO}$ ), vehicle alone, or siGLO red transfection indicator (Dharmacon) using a Lipofectamine RNAiMAX transfection kit (13778030; Invitrogen). After 48 hours of incubation at $37^{\circ} \mathrm{C}$ with $\mathrm{D} 3$ medium, the transfected cells were incubated with $0.4 \mu \mathrm{M}$ AF647-ApoA-I in $500 \mu \mathrm{l}$ of Dulbecco's modified Eagle's medium containing $0.1 \% \mathrm{w} / \mathrm{v}$ bovine serum albumin for 30 minutes at $37^{\circ} \mathrm{C}$. Then, $20 \mu \mathrm{g}$ of human serum transferrin (TRF) labeled with fluorescein isothiocyanate (FITC) (T2871; Invitrogen) was added to the dish, and the cell monolayer was incubated for an additional 30 minutes at $37^{\circ} \mathrm{C}$. The cells were washed, fixed, mounted, and imaged as described previously.

From the confocal micrographs, the intracellular fluorescence intensities of 25 cells for each treatment group $(n=4)$ were quantified using ImageJ software (National Institutes of Health, Bethesda, MD). To verify the siRNA knockdown of clathrin heavy chain, hCMEC/D3 cells were cultured on six-well plates (Corning, Corning, NY) and transfected with clathrin heavy chain siRNA or vehicle alone. After 48 hours, whole cell lysates were obtained and later assessed by Western blot.

Transcytosis Studies. The hCMEC/D3 cells were cultured on 12-mm Transwell filters with $0.4 \mu \mathrm{m}$ pores (Corning) as described elsewhere (Swaminathan et al., 2017). Upon reaching confluency, the polarized monolayer of hCMEC/D3 cells was incubated with $0.4 \mu \mathrm{M}$ AF647-ApoA-I in $500 \mu \mathrm{l}$ of D3 medium containing 1\% v/v FBS in the donor (luminal) compartment for 1 hour at $37^{\circ} \mathrm{C}$. The monolayer was washed with DPBS and fixed in ice-cold 4\% v/v PFA for 1 hour. The fixed cells were washed with DPBS and mounted as described earlier.

Alternately, hCMEC/D3 cells were cultured on 24-mm Transwell filters with $0.4 \mu \mathrm{m}$ pores. Upon reaching confluency, the cells were treated with AF647-ApoA-I as described earlier. At the end of the experiment, whole-cell lysates were obtained, and the solution in the receiver (abluminal) compartment was collected for subsequent detection of ApoA-I by Western blot.

Western Blot. Total protein levels in the whole cell lysates were quantified by bicinchoninic acid (BCA) assay using a commercial kit (Pierce, Waltham, MA). Equal protein quantities of each lysate were loaded onto a 4\%-12\% Criterion XT precast gel (Bio-Rad Laboratories, Hercules, CA). To detect ApoA-I protein in the abluminal solution obtained from the transcytosis studies, equal volumes of the abluminal solution collected at the end of the experiment were loaded onto the gel. The protein bands were electrotransferred onto a nitrocellulose membrane (Bio-Rad Laboratories). The blot was blocked for 1.25 hours with 5\% nonfat milk in Tris-buffered saline containing $0.1 \%$ Tween 20 (TBST), followed by overnight incubation at $4^{\circ} \mathrm{C}$ with the appropriate primary antibody solutions: glyceraldehyde-3-phosphate dehydrogenase (GAPDH) (1:1000, D16H11; Cell Signaling Technology, Danvers, MA), clathrin heavy chain (1:1000, D3C6; Cell Signaling Technology), or ApoA-I (1:1000, 5F4; Cell Signaling Technology).

The next day, the blot was washed 4 times with TBST and then incubated for 1.25 hours with the appropriate anti-rabbit or antimouse horseradish peroxidase-conjugated secondary antibody (1:5000; Cell Signaling Technology). After washing 4 times with TBST, the blot was incubated with SuperSignal West Dura Extended
Duration Substrate (ThermoFisher Scientific) and then analyzed by autoradiography using a CanoScan LiDE 110 film developer (Canon, Tokyo, Japan). The signal intensities of the protein bands were quantified by densitometry using ImageJ software.

Data Analysis. The plasma concentration of ${ }^{125} \mathrm{I}-\mathrm{ApoA}-\mathrm{I}$ from 0 to 59 minutes after injection was plotted as a function of time. To serve as a reference for comparison of ApoA-I plasma kinetics reported previously, data points were extracted from the study in which an intravenous bolus of Alexa Fluor 647-labeled recombinant ApoA-I (AF647-rApoA-I) was administered to plasma in mice (Stukas et al., 2014a). Based on the biexponential plasma concentration versus time profile of AF647-rApoA-I reported in that study, plasma concentration values from 0 to 3 hours corresponding to the initial phase were compared with those obtained for ${ }^{125}$ I-ApoA-I from 0 to 59 minutes in the present study.

The residual plasma volume $\left(V_{\mathrm{p}}\right)$ and permeability-surface area product (PS) for radioiodinated ApoA-I in each brain region were determined using methods described elsewhere (Poduslo and Curran, 1992). The $V_{\mathrm{p}}$ (microliters per gram) of each brain region was determined as follows:

$$
V_{\mathrm{p}}=\frac{q_{\mathrm{p}} \times 10^{3}}{C_{v} \times W}
$$

where $q_{\mathrm{p}}$ is the ${ }^{131} \mathrm{I}$-ApoA-I activity (counts per minute) in the brain region, $C_{v}$ is the concentration of ${ }^{131} \mathrm{I}$-ApoA-I (counts per minute per milliliter) in the plasma at 60 minutes, and $W$ is the weight (grams) of the brain region.

Given the total ${ }^{125} \mathrm{I}-\mathrm{ApoA}-\mathrm{I}$ activity in each brain region $\left(q_{\mathrm{T}}\right)$ (counts per minute), the ${ }^{125}$ I-ApoA-I activity in the extravascular space $(q)$ (counts per minute per gram) was determined as follows:

$$
q=\frac{q_{\mathrm{T}}}{W}-\frac{V_{\mathrm{p}} C_{a}}{10^{3}}
$$

where $C_{a}$ is the concentration of ${ }^{125} \mathrm{I}$-ApoA-I (counts per minute per milliliter) at $t=60$ minutes, corrected for spillover between ${ }^{125} \mathrm{I}$ and ${ }^{131}$ I channels.

The PS value (milliliters per gram per second) for ${ }^{125} \mathrm{I}-\mathrm{ApoA}-\mathrm{I}$ at each brain region was determined as follows:

$$
\mathrm{PS}=\frac{q}{\int_{0}^{t} C_{\mathrm{p}} d t}
$$

where $t$ is the circulation time (seconds), $q$ is the extravascular amount of ${ }^{125}$ I-ApoA-I (counts per minute per gram) in the brain region at time $t$, and $\int_{0}^{t} C_{\mathrm{p}} d t$ is the ${ }^{125} \mathrm{I}$-ApoA-I plasma area under the curve (AUC) for the time interval, 0-59 minutes. The plasma AUC (minutes $\times$ counts per minute/milliliters) of ${ }^{125}$ I-ApoA-I was calculated using the logarithmic trapezoidal method.

The overall PS value for ${ }^{125} \mathrm{I}-\mathrm{ApoA}-\mathrm{I}$ in rat brain was determined based on the total extravascular ${ }^{125}$ I-ApoA-I activity in all six brain regions, and was calculated in similar fashion. For comparison, the PS value for AF647-rApoA-I in mouse brain was determined using data extracted from the study by Stukas et al. (2014a) and was calculated by dividing the reported AF647-rApoA-I amount in brain at 1 hour (nanograms per milligram) by the plasma AUC from 0 to 1 hour $(\mathrm{h} \times \mu \mathrm{g} / \mathrm{ml})$ determined using the logarithmic trapezoidal method.

Statistical Analysis. All statistical tests were conducted using GraphPad Prism (GraphPad Software, La Jolla, CA). The statistical significance of differences observed in the slopes of the plasma concentration versus time curves for ${ }^{125}$ I-ApoA-I and AF647-rApoA-I was evaluated by F-test. The statistical significance of differences in the PS and $V_{\mathrm{p}}$ values determined for ${ }^{125} \mathrm{I}$-ApoA-I in different brain regions was evaluated by one-way ANOVA followed by Bonferroni post tests. The statistical significance of differences in the median fluorescence uptake of cells treated with/without $\mathrm{M} \beta \mathrm{CD}$ or nystatin was evaluated by one-way ANOVA followed by Bonferroni post tests. The statistical significance of differences in the mean fluorescence 
uptake of individual cells transfected with/without clathrin siRNA was evaluated by Student's $t$ test. The statistical significance of differences in clathrin heavy chain expression of cells transfected with/without clathrin siRNA was evaluated by Student's $t$ test.

\section{Results}

In this study, ${ }^{125}$ I-ApoA-I permeability at various brain regions was used to identify the distribution patterns indicative of transport across the $\mathrm{BBB}$ and/or the BCSFB. In addition, the mechanism of AF647-ApoA-I endocytosis in human cerebral microvascular endothelial cell (hCMEC/D3) monolayers was examined to support the distribution analysis.

Plasma Pharmacokinetics of ${ }^{125}$ I-ApoA-I. After intravenous bolus injection, the plasma concentration of ${ }^{125} \mathrm{I}-\mathrm{ApoA}-\mathrm{I}$ in adult rats declined in a log-linear fashion over the first 60 minutes (Fig. 2). The plasma pharmacokinetic parameters of ${ }^{125}$ I-ApoA-I were calculated by fitting the log-concentration versus time profile to a monoexponential equation. The rate constant for the ${ }^{125} \mathrm{I}$-ApoA-I decline in plasma was determined to be $0.0043 \pm 0.0004$ minute $^{-1}(n=6)$. Notably, this rate was not significantly different from the initial rate of AF647rApoA-I decline in the plasma of adult mice determined using the data reported by Stukas et al. (2014a).

Brain Permeability of ${ }^{125}$ I-ApoA-I. Average PS and $V_{\mathrm{p}}$ values for ${ }^{125}$ I-ApoA-I in various brain regions such as the cortex, caudate putamen, hippocampus, thalamus, brain stem, and cerebellum are shown in Table 1 . The PS and $V_{\mathrm{p}}$ values of ${ }^{125} \mathrm{I}$-ApoA-I in the brain stem and cerebellum are significantly greater than those determined for other brain regions $(P<0.05)$. The periventricular brain regions-the thalamus and hypothalamus - are anatomically adjacent to the choroid ventricles that form the BCSFB. Notably, the PS value of ${ }^{125} \mathrm{I}-\mathrm{ApoA}-\mathrm{I}$ in the thalamus is not significantly different from that determined for the cortex, caudate putamen, or hippocampus. The total PS value for ${ }^{125} \mathrm{I}$-ApoA-I in all six brain regions was found to be $0.47 \pm 0.11(\mathrm{ml} / \mathrm{g}$ per second $\left.\times 10^{-6}\right)$. This is in agreement with the PS value for AF647-rApoA-I in mouse brain $\left(0.29 \times 10^{-6} \mathrm{ml} / \mathrm{g}\right.$ per second, Fig. 2 inset), determined using the data extracted from the study by Stukas et al. (2014a).

Trafficking of AF647-ApoA-I at the BBB Endothelium. We conducted a battery of mechanistic assays to corroborate the in vivo findings and to further verify ApoA-I trafficking at the BBB. The Z-stack composite confocal micrographs of polarized hCMEC/D3 endothelial cell monolayers incubated with AF647-ApoA-I on the luminal (blood) side demonstrated AF647-ApoA-I internalization (Fig. 3A). Upon closer examination, tracts of AF647-ApoA-I moving to the abluminal (brain) side of the endothelium were clearly evident (Fig. 3B). Moreover, after AF647-ApoA-I incubation on the luminal side, full-length ApoA-I was detected by Western blot in both the cell lysate and abluminal solution, thus providing confirmation of its luminal-to-abluminal transcytosis (Fig. 3, C and D).

Mechanisms of AF647-ApoA-I Endocytosis in hCMEC/D3 Monolayers. Endocytosis is the predominant mechanism by which large proteins are internalized at the BBB endothelium (Xiao and Gan, 2013). Hence, we sought to investigate the mechanisms of AF647-ApoA-I endocytosis in hCMEC/D3 endothelial cells by use of the approaches of small-molecule inhibition and siRNA knockdown.

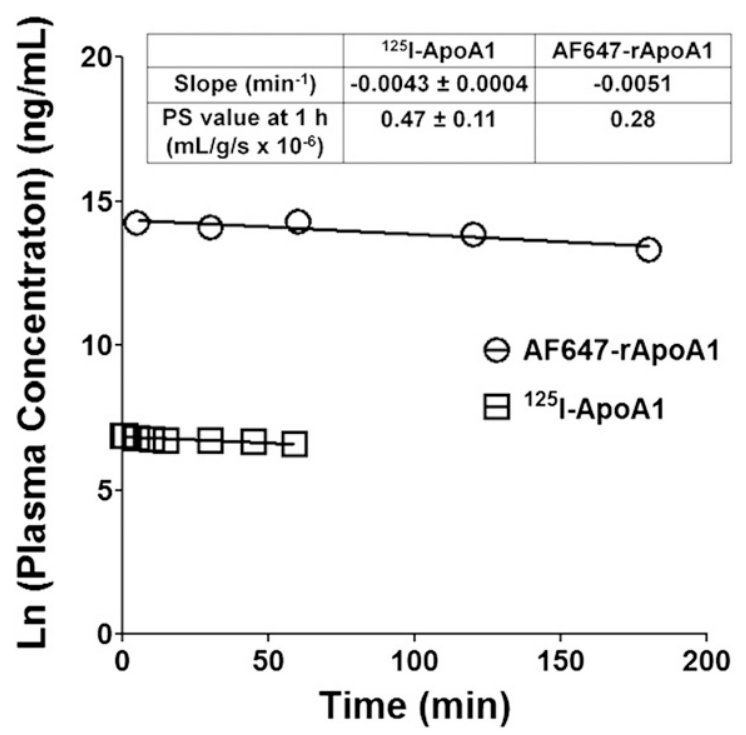

Fig. 2. Comparison of plasma concentration versus time profiles of ${ }^{125} \mathrm{I}-\mathrm{ApoA}-\mathrm{I}$ in rats $(\square)$ and Alexa Fluor 647-labeled recombinant ApoA-I (AF647-rApoA-I) in mice (O). Mouse data were extracted from a previous study by Stukas et al. (2014a) in which an intravenous bolus of AF647-rApoA-I $(60 \mathrm{mg} / \mathrm{kg})$ was injected via the tail vein ( $n=$ variable for each data point). Data points from the current study represent mean values ( $n=6$ for rats). Based on the F-test, the slope values are not significantly different: $\mathrm{F}(1,9)=0.1544, P=0.7035$. Slope and PS values for ${ }^{125} \mathrm{I}$-ApoA-I represent mean \pm S.D. $(n=6)$.

MBCD and Nystatin Reduce AF647-ApoA-I Uptake in hCMEC/D3 Monolayers. The uptake of AF647-ApoA-I by hCMEC/D3 monolayers with and without pretreatment with $\mathrm{M} \beta \mathrm{CD}$ or nystatin was investigated to evaluate the role of membrane cholesterol in AF647-ApoA-I endocytosis. The $\mathrm{M} \beta \mathrm{CD}$ is known to deplete membrane cholesterol whereas nystatin is known to sequester membrane cholesterol. Both of these agents are widely used to investigate cholesteroldependent endocytic pathways, often to study the involvement of lipid rafts (Zidovetzki and Levitan, 2007; Hussain et al., 2011). When evaluated by flow cytometry, AF647-ApoA-I uptake in hCMEC/D3 monolayers was significantly reduced after pretreatment with $10 \mathrm{mM} \mathrm{M} \beta \mathrm{CD}$ or $50 \mu \mathrm{M}$ nystatin $(P<0.001)$ (Fig. 4, $\mathrm{A}$ and $\mathrm{B}$ ). In addition, confocal micrographs of $\mathrm{M} \beta \mathrm{CD}$ treated hCMEC/D3 cell monolayers demonstrated lower intracellular accumulation of AF647-ApoA-I and showed bright fluorescence on the plasma membrane (Fig. 4C).

Role of Clathrin-Mediated Endocytosis in AF647ApoA-I Uptake by hCMEC/D3 Monolayers. The siRNA knockdown of clathrin heavy chain is expected to reduce the clathrin-mediated endocytosis of proteins. Transfection with siGLO red transfection indicator, a fluorescent oligonucleotide duplex, established the efficient transfection of siRNA in hCMEC/D3 monolayers (data not shown). The uptake of AF647-ApoA-I by clathrin siRNA transfected hCMEC/D3 monolayers was not notably altered (Fig. 5, A and B). However, the uptake of FITC-TRF was reduced in clathrin siRNA transfected monolayers, whereas the cells transfected with vehicle alone displayed punctate intracellular localization of FITC-TRF, possibly in the endosomes (Fig. 5, A and B).

The TRF is predominantly internalized at clathrin-coated pits and may thereby serve as a marker to investigate clathrinmediated endocytosis (Boucrot et al., 2006; McMahon and Boucrot, 2011; Mayle et al., 2012). Quantification of the confocal 
TABLE 1

The permeability-surface area product (PS) and cerebrovascular plasma volume $\left(V_{\mathrm{p}}\right)$ of ${ }^{125} \mathrm{I}$-ApoA-I in various regions of rat brain at 60 minutes Data represent mean \pm S.D. $(n=6) .{ }^{131} \mathrm{I}$-ApoA-I was injected to serve as a measure of $V_{\mathrm{p}}$. According to one-way ANOVA followed by Bonferroni post tests, the PS and $V_{\mathrm{p}}$ values for the brain stem and cerebellum are significantly greater $(P<0.05)$ than that of other regions.

\begin{tabular}{lcc}
\hline \multicolumn{1}{c}{ Brain Region } & PS & \multicolumn{1}{c}{$V_{\mathrm{p}}$} \\
\hline & $m l / g$ per second $\times 10^{-6}$ & $\mu l / g$ \\
Cortex & $0.39 \pm 0.08$ & $6.93 \pm 0.59$ \\
Caudate putamen & $0.28 \pm 0.07$ & $6.49 \pm 0.93$ \\
Hippocampus & $0.28 \pm 0.07$ & $6.94 \pm 0.84$ \\
Thalamus & $0.36 \pm 0.12$ & $9.45 \pm 1.27$ \\
Brain stem & $0.69 \pm 0.22$ & $14.37 \pm 2.50$ \\
Cerebellum & $0.76 \pm 0.21$ & $17.18 \pm 3.38$ \\
\hline
\end{tabular}

micrographs revealed that the intracellular fluorescence of FITC-TRF decreased significantly, but no change in AF647ApoA-I fluorescence was observed in siRNA transfected cells compared with cells transfected with vehicle alone $(P<0.001)$ (Fig. 5C). The decrease in expression of clathrin heavy chain after siRNA transfection was verified by Western blots (Fig. 5, $\mathrm{D}$ and $\mathrm{E})$.

\section{Discussion}

ApoA-I in the brain is expected to originate from the systemic circulation and therefore must undergo transport at the $\mathrm{BSCFB}$ and/or the $\mathrm{BBB}$. Upon intravenous bolus injection, AF647-rApoA-I fluorescence signal primarily localized at the choroid plexus, which led the investigators to conclude that BCSFB is the main portal for ApoA-I entry into brain from systemic circulation (Stukas et al. (2014a). Based solely on this observation, the uptake of ApoA-I via the BBB endothelium, which is a major portal for protein delivery to brain (Brasnjevic et al., 2009), cannot be ruled out. Moreover, due to the emerging role of ApoA-I in reducing $\mathrm{A} \beta$ deposition in the cerebral vasculature (CAA), clarifying the interaction and transcytosis of ApoA-I at the BBB is important for understanding the cerebrovascular contributions to $\mathrm{AD}$ pathogenesis.

To this end, we determined the PS of ${ }^{125} \mathrm{I}-\mathrm{ApoA}-\mathrm{I}$ in various brain regions after a femoral vein intravenous bolus injection in wild-type rats. The PS value is known to reflect permeability and thereby can be used to assess the relative contributions of the BBB and BCSFB in ApoA-I transport, based on the permeability differences observed at different brain regions (Smith and Rapoport, 1986). The total PS value of ${ }^{125} \mathrm{I}-\mathrm{ApoA}-\mathrm{I}$ in the entire rat brain coincided with the PS value of AF647-rApoA-I in mice determined from the data published by Stukas et al. (2014a). The lack of dependence on label and animal species may suggest broader applicability. Moreover, the plasma pharmacokinetics of ${ }^{125} \mathrm{I}-\mathrm{ApoA}-\mathrm{I}$ in the initial phase closely matched with that of AF647-rApoA-I. This agreement suggests that broader conclusions related to ApoA-I disposition in the plasma and brain could be drawn by employing the data from both studies.

The PS values of ${ }^{125}$ I-ApoA-I were found to vary with the brain region. Notably, the PS values in the brain stem and cerebellum were significantly higher compared with other regions. Although the physiologic relevance of these differences is not immediately apparent, it is important to stress that the PS value in the thalamus, which is anatomically adjacent to the ventricles, is not significantly higher compared with other brain regions.

Inorganic ions such as ${ }^{36} \mathrm{Cl}$ are predominantly transported into the brain via the $\mathrm{BCSFB}$, and have been reported to display preferential accumulation in thalamus (Smith and Rapoport, 1986). The moderate PS observed for ${ }^{125} \mathrm{I}-\mathrm{ApoA}-\mathrm{I}$ in thalamus relative to other regions suggests that ApoA-I transport at the BCSFB accounts for only a portion of the total ApoA-I brain delivery; therefore, the contribution of the $\mathrm{BBB}$ cannot be ignored. Moreover, the PS values observed for all brain regions are consistent with substantial ${ }^{125}$ I-ApoA-I transport across the entire diffuse network of cerebral microvessels that are lined by the BBB. As acknowledged by the authors in the previous study, their fluorescencebased approach may have lacked the sensitivity to capture the diffuse signal from AF647-rApoA-I in the cerebral vasculature. The use of ${ }^{125}$ I-ApoA-I, which could be detected with greater sensitivity than AF647-rApoA-I, enabled the detection of low levels of ${ }^{125}$ I-ApoA-I uptake at different brain regions without substantial processing of the brain tissue.

Protein delivery to brain is predominantly receptor-mediated, as the barrier properties of the BBB endothelium and choroid epithelium greatly restrict diffusional and paracellular
A

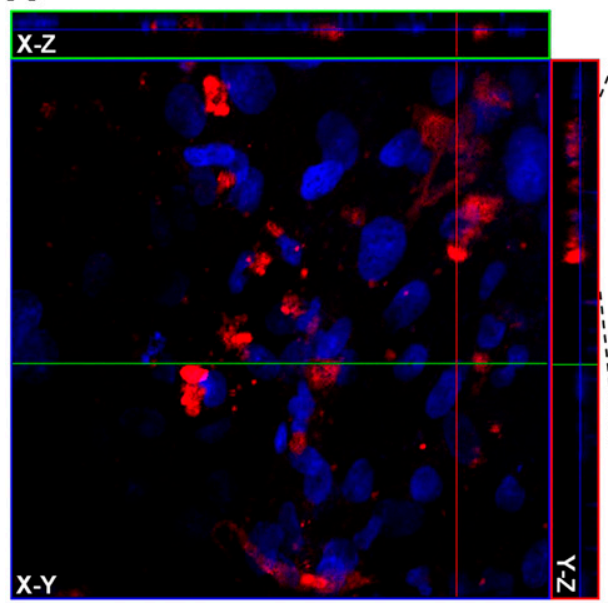

B

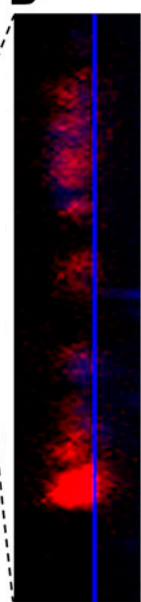

C

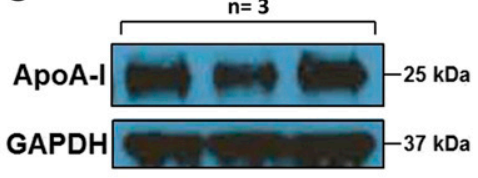

D

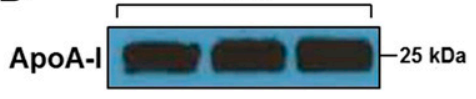

Fig. 3. Transcytosis of AF647-ApoA-I across hCMEC/D3 monolayers cultured on $0.4 \mu \mathrm{m}$ Transwell filters. (A) The Z-stack composite image demonstrates internalization of AF647-ApoA-I $(0.4 \mu \mathrm{M})$ in polarized hCMEC/D3 monolayers after 1 hour of incubation on the luminal side. The image presented in X-Y (transversal), $\mathrm{X}-\mathrm{Z}$ (vertical), and Y-Z (vertical) planes is a composite of 32 optical sections imaged with a $0.53 \mu \mathrm{m}$ Z-step interval. Red = AF647-ApoA-I; blue = DAPI-stained nuclei. (B) Enlarged section of the Y-Z plane showing permeation of AF647-ApoA-I across the endothelium. (C) Western blot showing ApoA-I in hCMEC/D3 lysate after 1 hour of incubation on the luminal side. (D) Western blot showing ApoA-I on the abluminal side after 1 hour of incubation on the luminal side. 
A

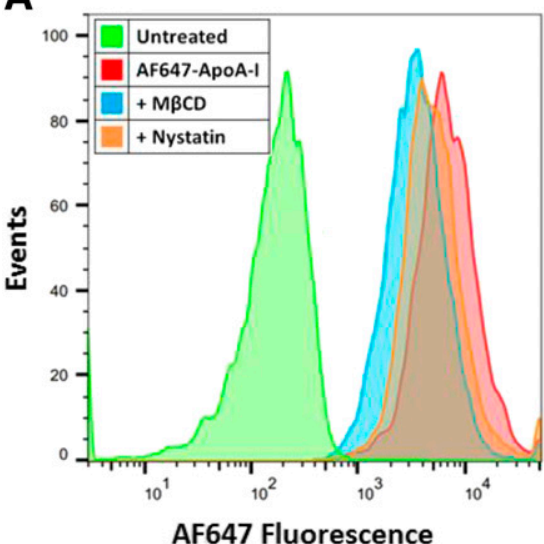

C

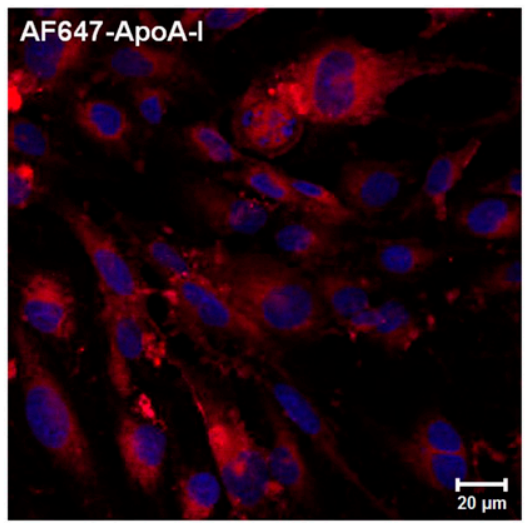

B
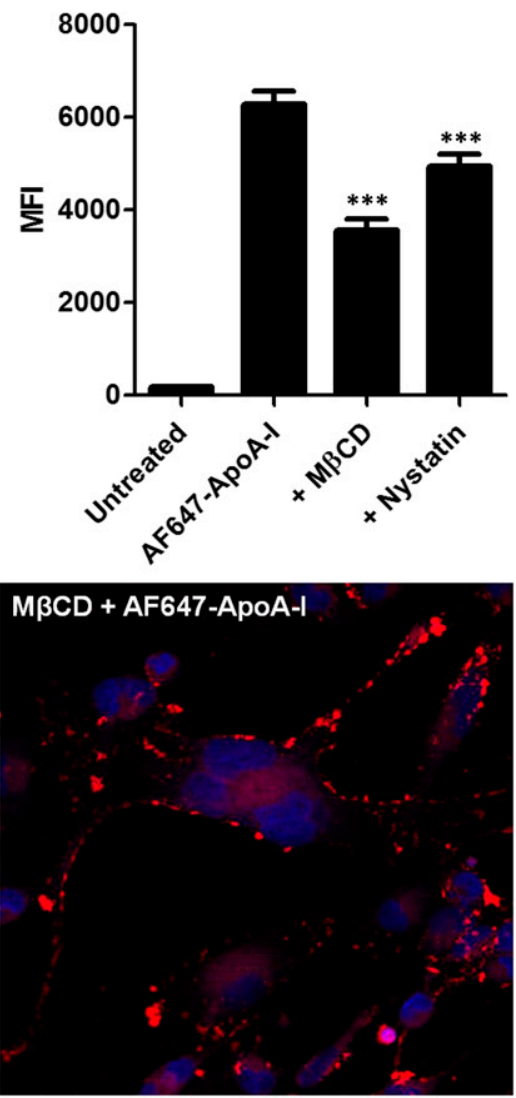

Fig. 4. Disruption of membrane cholesterol with methyl$\beta$-cyclodextrin $(\mathrm{M} \beta \mathrm{CD})$ or nystatin reduces the uptake of Alexa Fluor 647-labeled ApoA-I (AF647-ApoA-I) in hCMEC/D3 monolayers. (A) Representative flow cytometry histograms showing a decrease in the fluorescence uptake of AF647-ApoA-I $(0.4 \mu \mathrm{M})$ after 1 hour of pretreatment with $\mathrm{M} \beta \mathrm{CD}(10 \mathrm{mM})$ or Nystatin $(50 \mu \mathrm{M})$. (B) Evaluation by flow cytometry: fluorescence uptake of AF647-ApoA-I after pretreatment with $\mathrm{M} \beta \mathrm{CD}$ or nystatin represented as median fluorescence intensity (MFI) \pm S.D. $(n=3)$. One-way ANOVA followed by Bonferroni post tests showed a significant decrease in AF647-ApoA-I uptake in the $\mathrm{M} \beta \mathrm{CD}$ or nystatin pretreated cells compared with cells treated with AF647-ApoA-I alone $(* * * P<0.001)$. (C) Laser confocal micrographs of hCMEC/D3 monolayers pretreated with M $\beta C D$ show reduced intracellular accumulation of AF647-ApoA-I (right) when compared with cells treated with AF647ApoA-I alone (left). The images are representative of two independent experiments. Red = AF647-ApoA-I; blue = DAPI-stained nuclei. Scale bar, $20 \mu \mathrm{m}$. transport of macromolecules into the brain. Albumin and insulin are two endogenous proteins that are believed to exhibit receptor-mediated endocytosis at the BBB via gp60 and insulin receptors, respectively. However, Poduslo et al. (2001) have shown that the PS value of ${ }^{125} \mathrm{I}$-albumin in rat brain regions $\left(0.7-1.3 \mathrm{ml} / \mathrm{g}\right.$ per second $\left.\times 10^{-6}\right)$ is very low compared with that of ${ }^{125} \mathrm{I}$-insulin, which was determined to be between 14.0 and $20 \mathrm{ml} / \mathrm{g}$ per second $\times 10^{-6}$. The PS values of ${ }^{125} \mathrm{I}-\mathrm{ApoA}-\mathrm{I}$ in various brain regions, determined by similar approach, were found to be between 0.28 and $0.76 \mathrm{ml} / \mathrm{g}$ per second $\times 10^{-6}$, which are similar to that of ${ }^{125} \mathrm{I}$-albumin.

Previous studies have shown that luminal-to-abluminal transport of ApoA-I/HDL was saturable in porcine brain capillary endothelial cell (pBCEC) monolayers (Balazs et al., 2004). The lower PS values of ${ }^{125}$ I-ApoA-I could be due to receptor saturation by substantially higher ApoA-I plasma levels ( $\sim 7 \mathrm{mg} / \mathrm{dl})$ (Schonfeld et al., 1976). Despite low overall brain PS values, ApoA-I displays a very long plasma elimination half-life, which was reported to be 10.9 hours in mice (Stukas et al., 2014a). For comparison, the half-life of insulin was found to be around 10 minutes in mice (Cresto et al., 1977). Although the PS values of ${ }^{125}$ I-ApoA-I are 30 -fold lower than ${ }^{125} \mathrm{I}$-insulin, the plasma residence time of ${ }^{125} \mathrm{I}$-ApoA-I is $~ 60$-fold longer than that of ${ }^{125} \mathrm{I}$-insulin, which may make the overall brain uptake after a single dose injected in plasma similar for both proteins.

Using laser confocal microscopy, we have shown that AF647ApoA-I endocytosis in polarized hCMEC/D3 monolayers is cholesterol dependent. In addition, the Z-stack composite image of the internalized AF647-ApoA-I displayed a typical transcytosis pattern comparable to several other proteins we have studied previously that have well-characterized transport mechanisms at the BBB (Agyare et al., 2013; Swaminathan et al., 2017). By Western blot analysis, we also have shown that intact ApoA-I is transcytosed across the endothelial cell monolayer to the abluminal side.

Two previous studies have also demonstrated the luminalto-abluminal transcytosis of ApoA-I or HDL across in vitro BBB models (Balazs et al., 2004; Merino-Zamorano et al., 2016). A recent study showed the involvement of scavenger receptor class B type 1 (SR-B1) in the internalization of $\mathrm{HDL}$ in brain microvascular endothelial cells, which was found to be independent of both caveolin and clathrin (Fung et al., 2017). The investigators found that the internalized HDL colocalized with SR-B1, and that SR-B1 knockdown significantly reduced HDL uptake. Given that ApoA-I is the major protein component of plasma HDL, these findings suggest that SR-B1 at the BBB is responsible for ApoA-I endocytosis on the luminal side. The investigators also observed a decrease in HDL uptake after treatment with nystatin, a known membrane cholesterol disrupter. Moreover, SR-B1 is wellestablished as the HDL receptor mediating uptake in the liver to facilitate reverse cholesterol transport (Ganesan et al., 2016). These reports are consistent with the current findings that demonstrated a reduction in AF647-ApoA1 uptake after nystatin or $\mathrm{M} \beta \mathrm{CD}$ treatment. Based on these published reports and the observations made in our present study, we speculate that ApoA-I endocytosis is mediated by SR-B1 localized in noncaveolae lipid raft microdomains present on the luminal surface of the BBB. 
A

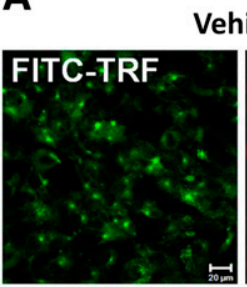

Vehicle
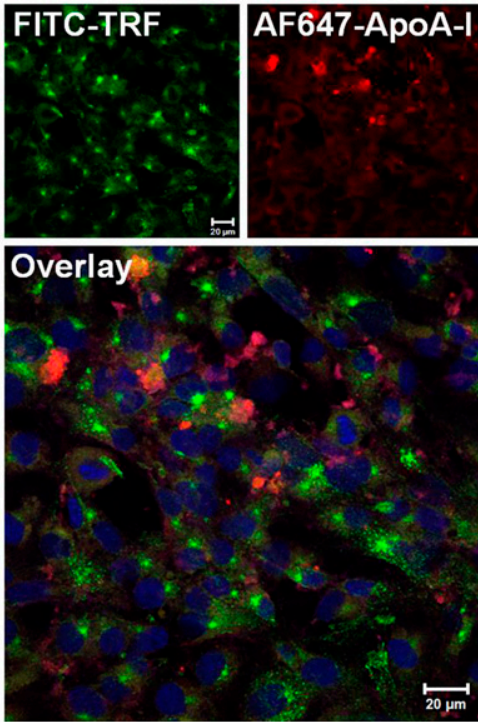

B
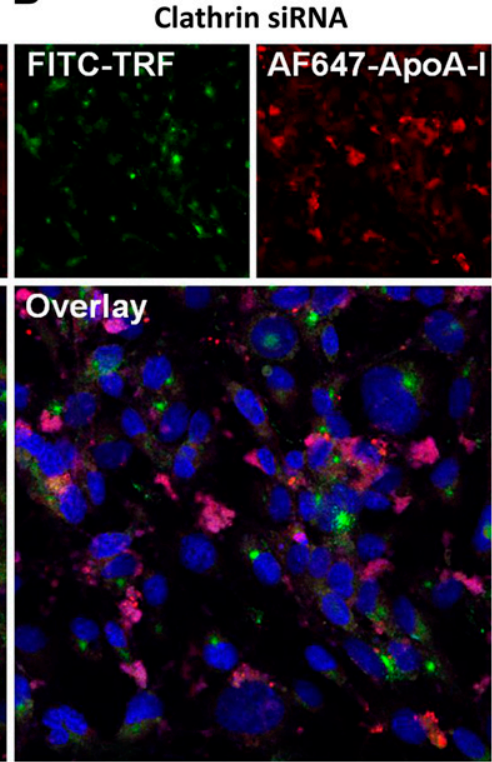

C

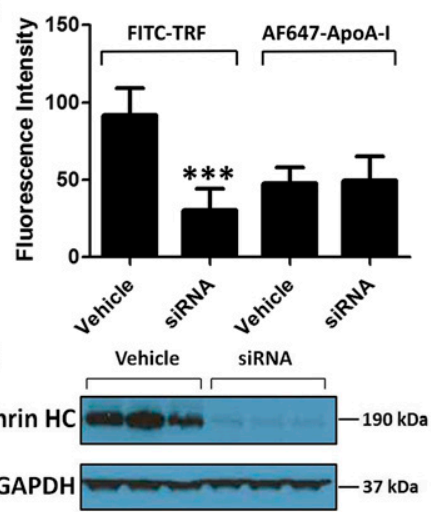

$\mathbf{E}$

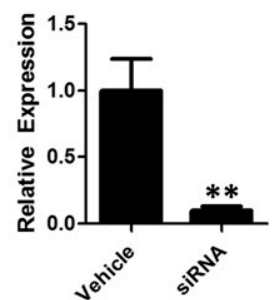

Fig. 5. Small interfering RNA knockdown of clathrin heavy chain has little effect on AF647-ApoA-I uptake in hCMEC/D3 monolayers. (A) Laser confocal micrographs of hCMEC/D3 transfected with vehicle alone show punctate localization of FITC-TRF, most likely in the endosomes. The images are representative of two independent experiments. (B) The hCMEC/D3 monolayers transfected with clathrin siRNA show greatly reduced uptake of FITC-TRF with little change in intracellular accumulation of AF647-ApoA-I $(0.4 \mu \mathrm{M})$. All images were processed similarly and obtained using the same instrument settings. Green = FITC-TRF; red = AF647-ApoA-I; blue = DAPI-stained nuclei. Scale bar, $20 \mu \mathrm{m}$. (C) Intracellular fluorescence intensities from 25 cells were quantified using ImageJ software and presented as mean \pm S.D. $(n=4)$. Student's $t$ test showed a significant decrease in FITC-TRF uptake with no change in AF647-ApoA-I uptake in the siRNA transfected cells compared with cells transfected with vehicle alone $(P<0.001)$ (D) Western blot showing the decrease in expression of clathrin heavy chain (HC) in hCMEC/D3 after siRNA transfection. (E) Semiquantitative analysis by densitometry showing the decrease in clathrin heavy chain expression in the siRNA transfected cells. Values are normalized to vehicle and presented as mean \pm S.D. $(n=3)$. Student's $t$ test showed a significant decrease in clathrin heavy chain expression for the siRNA transfected cells $(* * P<0.01)$.

Given the observed accumulation of AF647-ApoA1 in BBB endothelial cell monolayers, some portion of peripherally derived ApoA-I/HDL may exert its protective effect on the cerebral vasculature by accumulating in the BBB endothelium itself. Previous studies conducted to evaluate the role of ApoA-I overexpression/deletion on brain amyloid accumulation have shown that ApoA-I reduces cerebrovascular amyloid deposition with modest impact on parenchymal amyloid burden. A recent study demonstrated the ability of luminal ApoA-I to increase $\mathrm{A} \beta$ efflux across $\mathrm{BBB}$ endothelial cells from the abluminal side (Merino-Zamorano et al., 2016), suggesting that ApoA-I present in serum could influence brain clearance of $\mathrm{A} \beta$ in vivo. It is also possible that ApoA-I in the brain and plasma play differential roles in preserving cerebrovascular integrity and function.

In conclusion, we have shown that ApoA-I with ${ }^{125} \mathrm{I}$ or AF647 label are taken up at the BBB endothelium. The internalized ApoA-I could be used for endothelial-specific actions and/or may be transcytosed into brain parenchyma to elicit physiologic functions. Although our findings do not refute the brain delivery of ApoA-I via the BCSFB, they provide evidence to support the role of $\mathrm{BBB}$ in delivering ApoA-I to brain parenchyma and stress the need for further studies to resolve the mechanisms of cerebrovascular versus parenchymal actions of ApoA-I and its functional impact on the pathogenic processes of $\mathrm{CAA}$ and $\mathrm{AD}$.

\section{Acknowledgments}

We thank Dr. Timothy Wiedmann for his critical revisions to the manuscript.

\section{Authorship Contributions}

Participated in research design: Zhou, Swaminathan, Poduslo, Li, Kandimalla.

Conducted experiments: Zhou, Curran, Kandimalla, Lowe.

Contributed new reagents or analytic tools: Zhou, Curran, Lowe.

Performed data analysis: Zhou, Kandimalla.

Wrote or contributed to the writing of the manuscript: Zhou, Swaminathan, Li, Kandimalla.

\section{References}

Agyare EK, Leonard SR, Curran GL, Yu CC, Lowe VJ, Paravastu AK, Poduslo JF, and Kandimalla KK (2013) Traffic jam at the blood-brain barrier promotes greater accumulation of Alzheimer's disease amyloid- $\beta$ proteins in the cerebral vasculature. Mol Pharm 10:1557-1565.

Assmann G and Gotto AM Jr (2004) HDL cholesterol and protective factors in atherosclerosis. Circulation 109(23 Suppl 1):III8-III14.

Balazs Z, Panzenboeck U, Hammer A, Sovic A, Quehenberger O, Malle E, and Sattler W (2004) Uptake and transport of high-density lipoprotein (HDL) and HDL-associated alpha-tocopherol by an in vitro blood-brain barrier model. $J$ Neurochem 89:939-950.

Boucrot E, Saffarian S, Massol R, Kirchhausen T, and Ehrlich M (2006) Role of lipids and actin in the formation of clathrin-coated pits. Exp Cell Res 312: 4036-4048.

Brasnjevic I, Steinbusch HW, Schmitz C, and Martinez-Martinez P; European NanoBioPharmaceutics Research Initiative (2009) Delivery of peptide and protein drugs over the blood-brain barrier. Prog Neurobiol 87:212-251.

Cresto JC, Lavine RL, Buchly ML, Penhos JC, Bhathena SJ, and Recant L (1977) Half life of injected 125I-insulin in control and ob/ob mice. Acta Physiol Lat Am 27:7-15.

Elliott DA, Weickert CS, and Garner B (2010) Apolipoproteins in the brain: implications for neurological and psychiatric disorders. Clin Lipidol 51:555-573. Fung KY, Wang C, Nyegaard S, Heit B, Fairn GD, and Lee WL (2017) SR-BI mediated transcytosis of HDL in brain microvascular endothelial cells is independent of caveolin, clathrin, and PDZK1. Front Physiol 8:841.

Ganesan LP, Mates JM, Cheplowitz AM, Avila CL, Zimmerer JM, Yao Z, Maiseyeu A, Rajaram MVS, Robinson JM, and Anderson CL (2016) Scavenger receptor B1, the HDL receptor, is expressed abundantly in liver sinusoidal endothelial cells. Sci Rep 6:20646.

Hottman DA, Chernick D, Cheng S, Wang Z, and Li L (2014) HDL and cognition in neurodegenerative disorders. Neurobiol Dis $72(\mathrm{Pt} \mathrm{A}): 22-36$. 
Hussain KM, Leong KL, Ng MM, and Chu JJ (2011) The essential role of clathrinmediated endocytosis in the infectious entry of human enterovirus 71. J Biol Chem 286:309-321.

Kandimalla KK, Curran GL, Holasek SS, Gilles EJ, Wengenack TM, and Poduslo JF (2005) Pharmacokinetic analysis of the blood-brain barrier transport of 125Iamyloid beta protein 40 in wild-type and Alzheimer's disease transgenic mice (APP,PS1) and its implications for amyloid plaque formation. $J$ Pharmacol Exp Ther 313:1370-1378.

Koch M, Furtado JD, Falk K, Leypoldt F, Mukamal KJ, and Jensen MK (2017) Apolipoproteins and their subspecies in human cerebrospinal fluid and plasma. Alzheimers Dement (Amst) 6:182-187.

Lefterov I, Fitz NF, Cronican AA, Fogg A, Lefterov P, Kodali R, Wetzel R, and Koldamova R (2010) Apolipoprotein A-I deficiency increases cerebral amyloid angiopathy and cognitive deficits in APP/PS1 $\triangle \mathrm{E} 9$ mice. $J$ Biol Chem 285: 36945-36957.

Lewis TL, Cao D, Lu H, Mans RA, Su YR, Jungbauer L, Linton MF, Fazio S, LaDu MJ, and Li L (2010) Overexpression of human apolipoprotein A-I preserves cognitive function and attenuates neuroinflammation and cerebral amyloid angiopathy in a mouse model of Alzheimer disease. J Biol Chem 285: 36958-36968.

Mayle KM, Le AM, and Kamei DT (2012) The intracellular trafficking pathway of transferrin. Biochim Biophys Acta 1820:264-281.

McMahon HT and Boucrot E (2011) Molecular mechanism and physiological functions of clathrin-mediated endocytosis. Nat Rev Mol Cell Biol 12:517-533.

Merino-Zamorano C, Fernández-de Retana S, Montañola A, Batlle A, Saint-Pol J, Mysiorek C, Gosselet F, Montaner J, and Hernández-Guillamon M (2016) Modulation of amyloid- $\beta 1-40$ transport by ApoA1 and ApoJ across an in vitro model of the blood-brain barrier. J Alzheimers Dis 53:677-691.

Poduslo JF and Curran GL (1992) Increased permeability across the blood-nerve barrier of albumin glycated in vitro and in vivo from patients with diabetic polyneuropathy. Proc Natl Acad Sci USA 89:2218-2222.

Poduslo JF, Curran GL, and Berg CT (1994) Macromolecular permeability across the blood-nerve and blood-brain barriers. Proc Natl Acad Sci USA 91:5705-5709.

Poduslo JF, Curran GL, Wengenack TM, Malester B, and Duff K (2001) Permeability of proteins at the blood-brain barrier in the normal adult mouse and double transgenic mouse model of Alzheimer's disease. Neurobiol Dis 8:555-567.
Roheim PS, Carey M, Forte T, and Vega GL (1979) Apolipoproteins in human cerebrospinal fluid. Proc Natl Acad Sci USA 76:4646-4649.

Schonfeld G, Frick MS, and Bailey AP (1976) Measurement of apolipoprotein A-I in rat high density lipoprotein and in rat plasma by radioimmunoassay. $J$ Lipid Res 17:25-29.

Smith QR and Rapoport SI (1986) Cerebrovascular permeability coefficients to sodium, potassium, and chloride. $J$ Neurochem 46:1732-1742.

Strazielle N and Ghersi-Egea JF (2013) Physiology of blood-brain interfaces in relation to brain disposition of small compounds and macromolecules. Mol Pharm 10: 1473-1491.

Stukas S, Robert J, Lee M, Kulic I, Carr M, Tourigny K, Fan J, Namjoshi D, Lemke K, DeValle N, et al. (2014a) Intravenously injected human apolipoprotein A-I rapidly enters the central nervous system via the choroid plexus. J Am Heart Assoc 3:e001156 Stukas S, Robert J, and Wellington CL (2014b) High-density lipoproteins and cerebrovascular integrity in Alzheimer's disease. Cell Metab 19:574-591.

Swaminathan SK, Ahlschwede KM, Sarma V, Curran GL, Omtri RS, Decklever T, Lowe VJ, Poduslo JF, and Kandimalla KK (2017) Insulin differentially affects the distribution kinetics of amyloid beta 40 and 42 in plasma and brain. J Cereb Blood Flow Metab 38:904-918.

Weksler B, Romero IA, and Couraud PO (2013) The hCMEC/D3 cell line as a model of the human blood brain barrier. Fluids Barriers CNS 10:16.

Weller RO, Preston SD, Subash M, and Carare RO (2009) Cerebral amyloid angiopathy in the aetiology and immunotherapy of Alzheimer disease. Alzheimers Res Ther 1:6.

Xiao G and Gan LS (2013) Receptor-mediated endocytosis and brain delivery of therapeutic biologics. Int J Cell Biol 2013:703545.

Zidovetzki R and Levitan I (2007) Use of cyclodextrins to manipulate plasma membrane cholesterol content: evidence, misconceptions and control strategies. Biochim Biophys Acta 1768:1311-1324.

Address correspondence to: Dr. Karunya K. Kandimalla, University of Minnesota College of Pharmacy, Department of Pharmaceutics, Brain Barriers Research Center. 9-149A, Weaver-Densford Hall, 308 Harvard Street SE, Minneapolis, MN, 55455. E-mail: kkandima@umn.edu 